\title{
USO DA PLATAFORMA DE SIMULAÇÃO PHET NO PROCESSO DE ENSINO-APRENDIZAGEM DE MECÂNICA DOS FLUIDOS NO CURSO TÉCNICO EM QUÍMICA
}

DOI: $10.37702 / 2175-957 X . C O B E N G E .2021 .3452$

Aline Beatriz Pimentel Doelinger Oliveira - alinedoelinger@gmail.com

Instituto Federal do Espírito Santo

Rua João Bastos Vieira 185

29051-200 - Vitória - ES

ADRIANA ELAINE DA COSTA - AELCOSTA@HOTMAIL.COM

INSTITUTO FEDERAL DO ESPÍRITO SANTO IFES

RUA DOUTOR ANICETO FRIZZERA FILHO 10

29102-070 - VILA VELHA - ES

Estela Claudia Ferretti - estelaferretti@yahoo.com.br

Instituto Federal do Espírito Santo

Rua Inácio Higino 1170

29101-094 - Vila Velha - ES

FÁTIMA PERES ZAGO DE OLIVEIRA - fatima.oliveira@ifc.edu.br Instituto Federal Catarinense

Rua Eleonora Budag 71

89165-658 - Rio do Sul - SC

Resumo: $O$ presente artigo analisa e discute o uso de um aplicativo de simulação no ensino de Mecânica dos Fluidos, conteúdo da ementa da disciplina de Operações Unitárias, ministrada no quarto período de um curso Técnico em Química concomitante da Rede Federal. O trabalho é fruto da continuidade da pesquisa desenvolvida em projeto de Iniciação Científica, iniciado com a identificação de estilos de aprendizagem dos estudantes e continuado para implementar estratégias pedagógicas capazes de contemplar diversos perfis. A partir da busca e estudos do grupo de pesquisa, selecionou-se o módulo Pressão de um Fluido e Fluxo, disponível na plataforma gratuita PhET Interactive Simulations, utilizando a simulação Pressão, sobre estática dos fluidos, e a simulação Fluxo, sobre cinemática dos fluidos. Os estudantes foram avaliados 
quanto ao desempenho em questões fechadas propostas com o uso da plataforma, em que puderam explorar as ferramentas de medição, alterando propriedades do fluido e dos sistemas, realizando simulações, cálculos, analisando e comentando os efeitos observados em diferentes situações. Também foi proposta a eles a avaliação qualitativa da plataforma por meio de questões abertas. A partir da Análise Textual Discursiva, emergiram das falas dos estudantes as categorias contribuições da tecnologia no processo de ensino-aprendizagem e obstáculos presentes no uso da plataforma. Discutiu-se, nestas, sobre o desempenho dos estudantes, suas opiniões acerca do uso da plataforma e contribuições para o aprendizado, destacando-se a convergência entre os conhecimentos teóricos e sua aplicação em um sistema simulado e a aplicação como um recurso didático que torna a aula mais dinâmica.

Palavras-chave: Simulação. Ensino-Aprendizagem. Operações Unitárias. 


\section{USO DA PLATAFORMA DE SIMULAÇÃO PHET NO PROCESSO DE ENSINO-APRENDIZAGEM DE MECÂNICA DOS FLUIDOS NO CURSO TÉCNICO EM QUÍMICA}

\section{INTRODUÇÃO}

O avanço tecnológico vem impactando profundamente o modo de vida da sociedade contemporânea, e a educação não está alheia a essas transformações. No ensino de ciências exatas e tecnológicas, sobretudo, há um movimento pedagógico constante atrelado à tecnologia, buscando proporcionar aos estudantes e futuros profissionais um ensino que contemple as variáveis da contemporaneidade. Objetiva-se, também, com essa atualização, superar os paradigmas tradicionais de ensino, que muitas vezes dissociam o conhecimento escolar da realidade do aluno e das possibilidades que a era digital agrega ao ensino.

A disciplina de Operações Unitárias, ministrada no quarto período do Curso Técnico em Química do Instituto Federal do Espírito Santo (Ifes) - campus Vila Velha, busca integrar conhecimentos de áreas como matemática, química e física, além de assuntos que constam em cursos de Engenharia, como Mecânica dos Fluidos e Balanço de Massa e Energia, favorecendo que o futuro Técnico em Química tenha uma visão geral sobre a aplicação e a importância desses conceitos em seu cotidiano e no ambiente industrial. Por ser um curso da modalidade concomitante, o público atendido reúne desde estudantes que ainda estão cursando o ensino médio a estudantes que estão distantes do meio acadêmico e buscam uma qualificação a mais para se destacar no mundo do trabalho. Essa diversidade ocasiona um descompasso entre a dinâmica das aulas e o ritmo de aprendizagem dos estudantes, que encontram dificuldades na integração dos conhecimentos, tendo consequência a não aprovação na disciplina ou a evasão do curso. A fim de superar esse cenário, iniciou-se por meio de um projeto de Iniciação Científica a identificação dos estilos de aprendizagem dos estudantes do curso Técnico em Química (SCHAFFEL et al., 2019), e o trabalho foi continuado a fim de desenvolver estratégias pedagógicas capazes de contemplar o público heterogêneo do curso, tornando a disciplina mais inclusiva, almejando também o despertar de vocações para a Engenharia.

No conteúdo de Mecânica dos Fluidos, que possui intrínseca relação com os princípios da Física e da Matemática, os alunos apontam a dificuldade em estabelecer relações entre os fenômenos reais e as equações e cálculos desenvolvidos, sendo necessário pensar em estratégias que favoreçam essas relações, tornando a aprendizagem mais efetiva. Como alternativa, pensou-se inicialmente em utilizar simuladores para a experimentação virtual. O professor engenheiro geralmente sai da graduação programando e simulando em plataformas como Fortran e Matlab, que são muito avançadas para ensino de nível médio. Dessa forma, a partir de busca, seleção e estudo de plataformas com objetos de aprendizagem compatíveis com o nível técnico, implementou-se na disciplina, no ano de 2019, o uso do aplicativo de simulação Pressão de um Fluido e Fluxo, disponibilizado na plataforma PhET Interactive Simulations, que reúne aplicações das áreas das ciências exatas e biológicas, de forma gratuita e acessível (UNIVERSITY OF COLORADO BOULDER, 2021). O presente trabalho visa discutir os resultados do uso das simulações nas aulas de Mecânica dos Fluidos, suas contribuições para o processo de ensino-aprendizagem na disciplina e a percepção pedagógica do professor de Operações Unitárias. 


\section{REVISÃO BIBLIOGRÁFICA}

\subsection{Educação e desenvolvimento tecnológico}

As tecnologias podem favorecer a aprendizagem, em particular quando o aluno, com apoio do professor, é instigado a avançar progressivamente em situações e problemas, construindo assim a autonomia do estudante. Demo (2009) aponta a "multiplicidade de oportunidades de aprender" que o aluno pode encontrar em ambientes mediados por novas tecnologias, não incidindo em panacéias tecnológicas, mas considerando que estas oportunidades são mais flexíveis, motivadoras e centradas na atividade dos alunos. Por tais motivos, são potencialmente capazes de sustentar processos de autoria e autonomia.

No que toca aos alunos, o uso de recursos tecnológicos voltados à aprendizagem valoriza a iniciativa e condiz com sua realidade (ARANTES et al., 2010; PINHEIRO et al., 2012; SILVA; MELO, 2016), principalmente para os mais jovens, ditos nativos digitais. Observa-se que esta discussão não se estende à exclusão digital decorrente da desigualdade socioeconômica, mas admite-se que constitui um problema cujo enfrentamento é de caráter essencial e emergencial.

Quanto ao professor, seu papel é de orientar, avaliar, acompanhar, motivar, dialogar e questionar, como parceiro mais experiente que presta suporte, não oferecendo receitas prontas e tampouco abandonando os alunos em suas atividades. O professor aparece nesse cenário como quem ensina e aprende, sem a pretensão de saber tudo, mas compondo interesses, liderando processos, ativando dinâmicas, estimulando a construção de valores e elevando os desafios, com o compromisso de trazer para o aluno o que há de melhor no mundo do conhecimento e da tecnologia (DEMO, 2009; NEVES e TEODORO, 2013).

É interessante constatar que as novas tecnologias não inventaram o papel do professor mediador e nem a aprendizagem, mas reconstruíram heranças de teorias clássicas, como as relacionadas por Demo (2009): herança socrática da orientação docente que não oferece resposta pronta a dúvidas; herança freireana no professor que se orienta pelas experiências de vida dos alunos (FREIRE, 2006); herança piagetiana da equilibração através de etapas sucessivas e progressivas; herança autopoiética, na qual o aprendiz precisa se sentir mais do que executor, mas autor que constrói sua própria história (MATURANA, 2001); herança das teorias de aprendizagem significativa, que ressaltam a importância da significação dos conteúdos, isto é, o que os mesmos tenham sentido para o aprendiz e provoquem o seu engajamento, assumindo importância em sua vida e encaixando-se em propósitos próprios (AUSUBEL, 1968; AUSUBEL, 2000; MOREIRA, 2006).

Ao mesmo passo em que o bom uso guarda potencialidades, a mediação inadequada no uso de tecnologias pode levar a uma aprendizagem reduzida ou inexistente. Também é importante enfatizar que as novas tecnologias devem ser apoiadas por processos formativos que perpassem os aspectos técnicos, priorizando a vida, o desenvolvimento, a dignidade humana, o bem viver coletivo e a integridade do ambiente. Uma educação fundamentada na premissa da "tecnologia pela tecnologia" não sustenta a formação de profissionais de Engenharia que atuarão no âmbito de uma sociedade que carece de cidadãos comprometidos e responsáveis com os problemas coletivos (BORDIN; BAZZO, 2017). Muito além de desenvolver e aplicar soluções tecnológicas, é necessário que o Engenheiro estabeleça uma "atitude avaliativa e proativa de acordo com parâmetros e interesses coletivos" (BORDIN; BAZZO, 2018).

Moran (2013) destaca também a necessidade de capacitação e formação continuada para os professores e demais profissionais de uma instituição que pretende se aprofundar na integração entre tecnologia e aprendizagem. Essa formação deve abranger 
os aspectos técnicos e também pedagógicos, para que a equipe domine as ferramentas e como melhor empregá-las no cotidiano escolar.

\subsection{Objetos de aprendizagem no ensino de ciências exatas e tecnológicas}

Um objeto de aprendizagem (OA) é todo e qualquer recurso utilizado para potencializar o processo de ensino-aprendizagem. Em uma definição mais abrangente, os objetos de aprendizagem aparecem não só como recursos digitais e diferem de outras mídias que podem ser utilizadas como recursos no quesito de permitirem múltiplos e simultâneos acessos, por serem disponibilizados na internet. Isso possibilita aos estudantes que possam explorar melhor o recurso, tendo o professor como mediador do conhecimento o qual desejamos que o aluno se aproprie utilizando um OA (BRASIL, 2014).

No ensino de ciências exatas e tecnológicas, o uso de objetos de aprendizagem vem ganhando destaque e se revela como um grande aliado na superação das características do ensino tradicional que limitam o potencial dos estudantes, como o excesso de exposição oral e ausência de recursos que acompanhem as transformações da contemporaneidade. A exemplo, os objetos de aprendizagem categorizados como simuladores computacionais tornam possível ilustrar situações que são difíceis para serem demonstradas apenas com recursos didáticos tradicionais como quadro e pincel.

Existem diversas plataformas contendo aplicações de simulações, com diferentes níveis de complexidade, para diferentes níveis de ensino. No ensino de Engenharia, costuma-se utilizar softwares mais sofisticados como Fortran e Matlab, que inicialmente são muito avançados para o ensino de nível médio. A plataforma PhET Interactive Simulations surge como uma alternativa quando se busca por aplicações que abranjam o nível médio e também podem ser estendidas para o nível técnico, sem perder a qualidade conceitual. Às aplicações apresentam recursos dinâmicos, com design simples e que também são desenvolvidos com acessibilidade, para contemplar todos os públicos. Ademais, estão disponíveis no formato de aplicativo para executar no computador ou para embutir em outros sites.

Diversos autores já utilizam a plataforma PhET nas aulas de física, química e também biologia. Schmitt e Schein (2020) utilizaram a aplicação "Forças e Movimento" para o ensino de Cinemática, destacando a amplitude de conceitos a serem abordados e a possibilidade do protagonismo dos estudantes na exploração e experimentação da aplicação. Mardegan (2020) também relata as contribuições do uso da PhET como laboratório virtual de Física para o Ensino Médio, em que utilizou-se também as aplicações "Balançando" e "Laboratório do Pêndulo", especialmente na resolução de exercícios. Outra vantagem a ser destacada é que o momento de experimentação virtual por meio da plataforma $P h E T$ pode ser continuado pelo aluno em sua casa, indo além do espaço escolar e se tornando uma ferramenta complementar ao estudo de Mecânica dos Fluidos, uma vez que o acesso é gratuito e demanda poucos recursos, diferentemente de uma prática laboratorial convencional, cuja realização e discussão se restringe ao momento disponibilizado na rotina escolar. Destaca-se também a economia de recursos físicos empregados nos experimentos tradicionais.

\section{METODOLOGIA}

\subsection{Seleção da aplicação de simulação e coleta dos dados}

Um dos problemas geralmente mencionados na inserção de simulações em aulas do ensino médio muitas vezes decorre do tempo limitado para que o professor possa pesquisar e avaliar novas ferramentas para o ensino. Além disso, de acordo com Hargreaves e Fullan (2000), o isolamento profissional é um limitador do acesso a novas 
ideias e soluções e às mudanças necessárias à escola. De acordo com os autores, ocorre falta de oportunidades e de encorajamento para a atuação conjunta de professores, para a troca de aprendizado e, para melhorarem sua qualificação como comunidade, fatores impulsionados por problemas como conservadorismo e resistência à inovação no ensino, falta de apoio aos professores iniciantes, pressão por resultados imediatos por parte dos que elaboram as políticas, dentre outros motivos. No caso das disciplinas de Operações Unitárias e Fenômenos de Transporte, ministradas em cursos de nível médio e superior no Ifes - campus Vila Velha, sentiu-se necessidade da formação de um grupo cooperativo, a fim de romper com o planejamento isolado e de implementar novas propostas didáticas para a superação das dificuldades na compreensão de determinados conceitos pelos estudantes. O grupo formado é composto por duas professoras da área de Engenharia, por uma professora doutora na área de Educação Matemática, que proporciona suporte pedagógico e contribui no desenvolvimento das estratégias e por uma aluna de Iniciação Científica (IC) já formada no curso Técnico em Química, que atualmente cursa Licenciatura em Química.

Para o desenvolvimento deste trabalho, uma das professoras do grupo realizou uma busca, selecionando a plataforma PhET Interactive Simulations como fonte de aplicações de simulação, dadas as possibilidades existentes nessa plataforma. Selecionou-se o módulo Pressão de um Fluido e Fluxo, por ser perfeitamente compatível com assuntos que fazem parte da ementa da disciplina de Operações Unitárias do curso Técnico em Química, no conteúdo de Introdução à Mecânica dos Fluidos. Nesse módulo, foram selecionadas para desenvolvimento de atividade duas simulações: pressão de fluidos em sistemas estáticos e escoamento com vazão constante em duto cilíndrico. A aluna de IC estudou sobre o simulador por meio das instruções disponibilizadas pela plataforma e a professora regente das turmas elaborou exercícios conceituais para aplicação em sala com o auxílio da estudante de IC.

Devido ao tempo de duração da aula, de apenas 100 minutos, divididos entre a realização da atividade de simulação e a avaliação qualitativa da mesma, optou-se por elaborar um instrumento avaliativo na forma de questionário, que fosse de rápida aplicação e fácil compreensão por parte dos estudantes. Elaborou-se duas questões: a primeira, de múltipla escolha em cinco níveis, para que os estudantes pudessem avaliar o desempenho geral da plataforma e a segunda, uma questão aberta, para que estes pudessem descrever de que forma a simulação contribuiu para o entendimento dos conceitos teóricos previamente estudados. Os estudantes responderam ao questionário logo após a realização das simulações computacionais.

A coleta dos dados foi realizada nos dois semestres letivos do ano de 2019, envolvendo três turmas. Ao total, 68 alunos participaram das aulas realizadas em laboratório de informática, utilizando o módulo selecionado para a resolução dos exercícios elaborados. As aulas presenciais foram suspensas em março de 2020 para conter a disseminação do vírus Sars-Cov-2, causador da Covid-19, e o ensino remoto emergencial foi implementado em maio do mesmo ano para os cursos do campus.

Assim, diante das fragilidades evidenciadas pelo contexto pandêmico, a coleta dos dados foi interrompida devido ao fato de que a maioria dos estudantes do curso não possui condições de acesso à plataforma, uma vez que esse acesso é feito por computadores. Sem computadores nas suas residências e impossibilitados de ir ao campus para realizar as atividades, optou-se por suspender a dinâmica temporariamente para não causar prejuízos aos estudantes. 


\subsection{Análise dos dados}

Os dados levantados por intermédio das respostas à primeira questão do instrumento avaliativo, de múltipla escolha, foram submetidos à análise descritiva (BARBETTA, 2010), na qual as frequências de cada um dos cinco níveis de resposta foram dispostas em um gráfico apropriado.

No que se refere à segunda questão, do tipo aberta, os dados foram submetidos à análise textual discursiva (MORAES, 2016). Esse tipo de análise constitui-se em leitura profunda dos textos coletados, de modo que desses emerjam categorias de palavras ou expressões a serem analisadas, em um processo constante de construção e reconstrução de sentidos. Após a identificação e definição das categorias, as mesmas podem ser quantificadas.

Além da análise dos dados coletados pela aplicação do instrumento avaliativo, também foi considerada a percepção da professora regente da disciplina quanto à participação, motivação e desempenho dos estudantes durante as atividades de simulação computacional.

\section{RESULTADOS E DISCUSSÕES}

\subsection{Desempenho dos estudantes nas questões conceituais}

Anteriormente ao início da resolução de exercícios, a aluna de IC explicou o que era simulação, o ambiente da plataforma e as possibilidades de medição e variação das propriedades do fluido e do sistema. De acordo com a PhET Interactive Simulations, propõe-se, nessas simulações, que o aluno possa investigar como a pressão muda no ar e na água, descobrir como alterar a pressão, estimar a pressão numa variedade de situações, determinar como o movimento de um fluido afeta a pressão e observar como converter a pressão da água em velocidade da água.

Na simulação Pressão, utilizada para fluidos estáticos, a professora regente fez uma demonstração de aplicação selecionando a água como fluido no ambiente de simulação. Cada dupla de estudantes poderia escolher a escala de pressão, quantos blocos utilizar e a densidade do fluido (exceto água, que já havia sido usada no exemplo). Nas figuras 1 e 2, observa-se a interface gráfica da simulação Pressão.

Figura 1 - Tela inicial da simulação Pressão

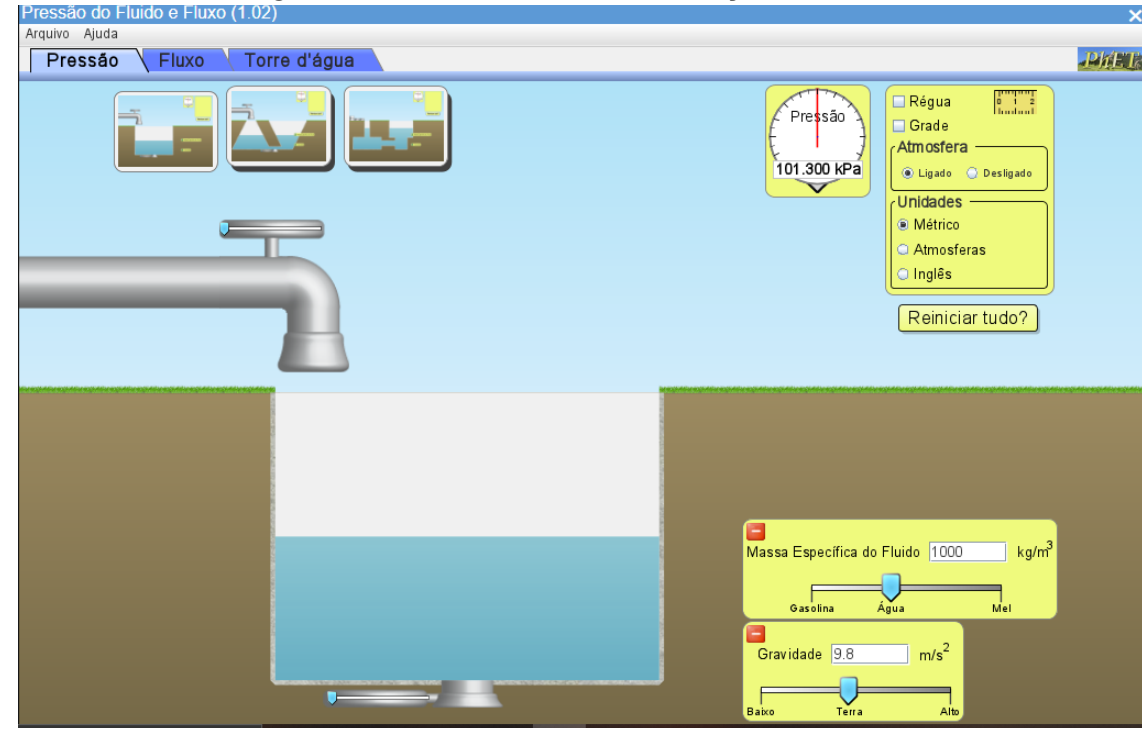

Fonte: PhET, 2021 
Explorando as ferramentas de medição da plataforma e alterando propriedades do fluido e dos sistemas, os estudantes foram orientados a realizar simulações e confirmar os resultados com seus cálculos, analisando e comentando os efeitos observados em diferentes situações. Com o apoio da professora durante a resolução dos exercícios, todos os estudantes alcançaram o resultado esperado nas questões conceituais sobre as simulações.

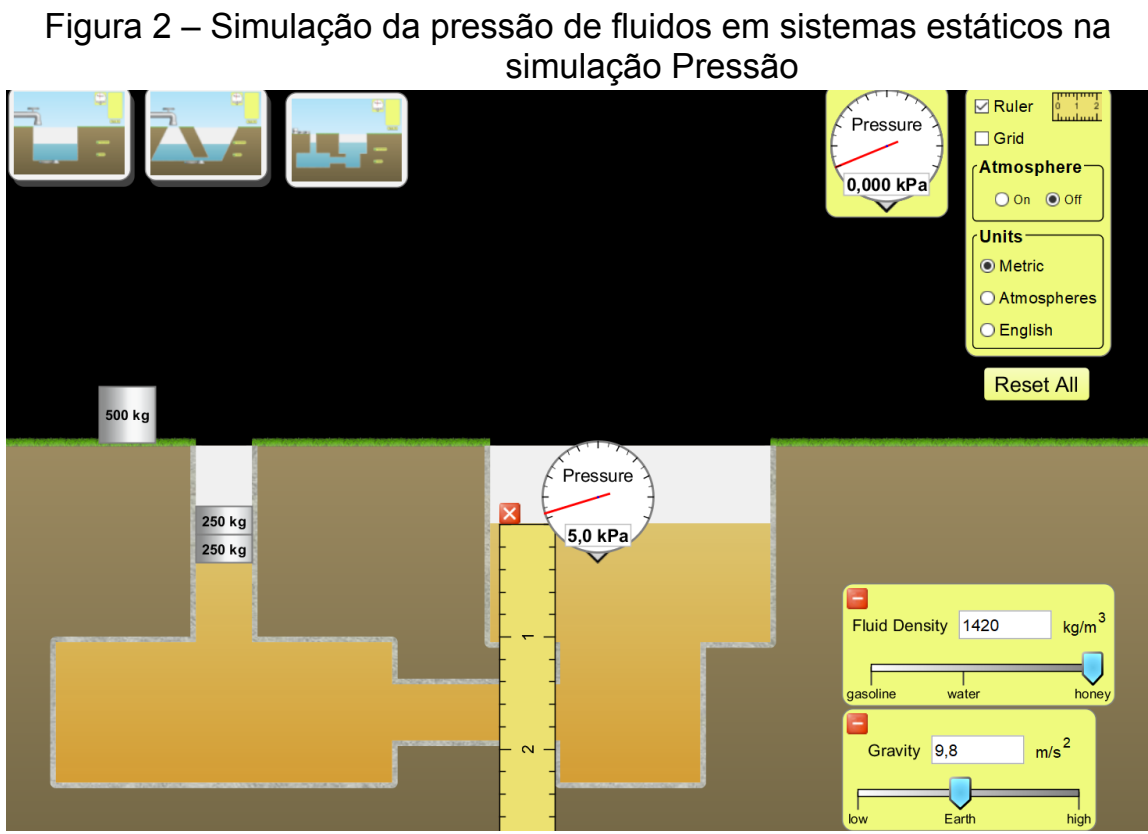

Fonte:acervo pessoal, 2019.

No Quadro 1 são apresentados os cálculos e comentários realizados pela dupla.

Quadro 1 - Cálculos e comentários da dupla de estudantes sobre a simulação apresentada na Figura 2

\begin{tabular}{|c|c|c|}
\hline Dados e Objetivos & Cálculos desenvolvidos & Comentários dos estudantes \\
\hline $\begin{array}{l}\text { Escala de pressão: Relativa. } \\
\text { Pressão atmosférica: 0,0 Pa. } \\
\text { Fluido: mel } \\
\text { Densidade: } 1420 \mathrm{~kg} / \mathrm{m} \\
\text { Aceleração da gravidade: } 9,8 \mathrm{~m} / \mathrm{s}^{2} \text {. } \\
\text { Número de blocos utilizados: } 02 . \\
\text { Massa dos blocos: } 250 \mathrm{~kg} \text { cada. } \\
\text { Objetivo: determinar o valor da } \\
\text { pressão no nível imediatamente } \\
\text { abaixo dos blocos; analisar o que } \\
\text { ocorre quando a densidade do } \\
\text { fluido varia. }\end{array}$ & $\begin{array}{l}P=P_{\text {atmosférica }}+\rho \cdot g \cdot h \\
P=0+1420 \cdot 9,8 \cdot 0,36 \\
P=5009,76 \mathrm{~Pa} \\
P \cong 5,0 \mathrm{KPa}\end{array}$ & $\begin{array}{l}\text { A cota } h \text { medida ao lado direito } \\
\text { do sistema é } 0,36 \mathrm{~m} \text {. } \\
\text { O valor calculado de pressão } \\
\text { ficou próximo do simulado. } \\
\text { Diminuindo a densidade do } \\
\text { líquido, sua pressão diminui e o } \\
\text { equilíbrio desloca-se da } \\
\text { esquerda para a direita (o nível } \\
\text { de líquido do lado esquerdo } \\
\text { diminui devido à pressão dos } \\
\text { blocos; o nível do lado direito } \\
\text { aumenta). }\end{array}$ \\
\hline
\end{tabular}

Fonte: acervo pessoal, 2019.

De modo geral, com simulações em diferentes condições, todas as duplas atingiram os objetivos da atividade. Duas dificuldades apontadas pelas duplas foram: a obtenção do valor de $h$ com duas casas decimais, devido à precisão da escala da régua de medição da plataforma; a obtenção de valor preciso de pressão, devido à baixa sensibilidade dos mouses em alguns computadores do laboratório de informática.

$\mathrm{Na}$ simulação Fluxo, referente à cinemática dos fluidos, foi possível variar o diâmetro das tubulações de escoamento em diferentes posições no sistema, a massa específica do fluido e a vazão. Destaca-se, nessa simulação, a possibilidade de visualizar alguns detalhes internos da movimentação do fluido, como as trajetórias do escoamento 
de algumas moléculas aleatórias do fluido com cores, o que não é possível visualizar em um experimento convencional. Assim, mesmo quando a experimentação real é possível, esse simulador continua sendo um complemento significativo. Na Figura 3 é apresentado o resultado de uma das duplas de estudantes sobre a simulação Fluxo.

Figura 3 - Simulação de escoamento com vazão constante em duto cilíndrico

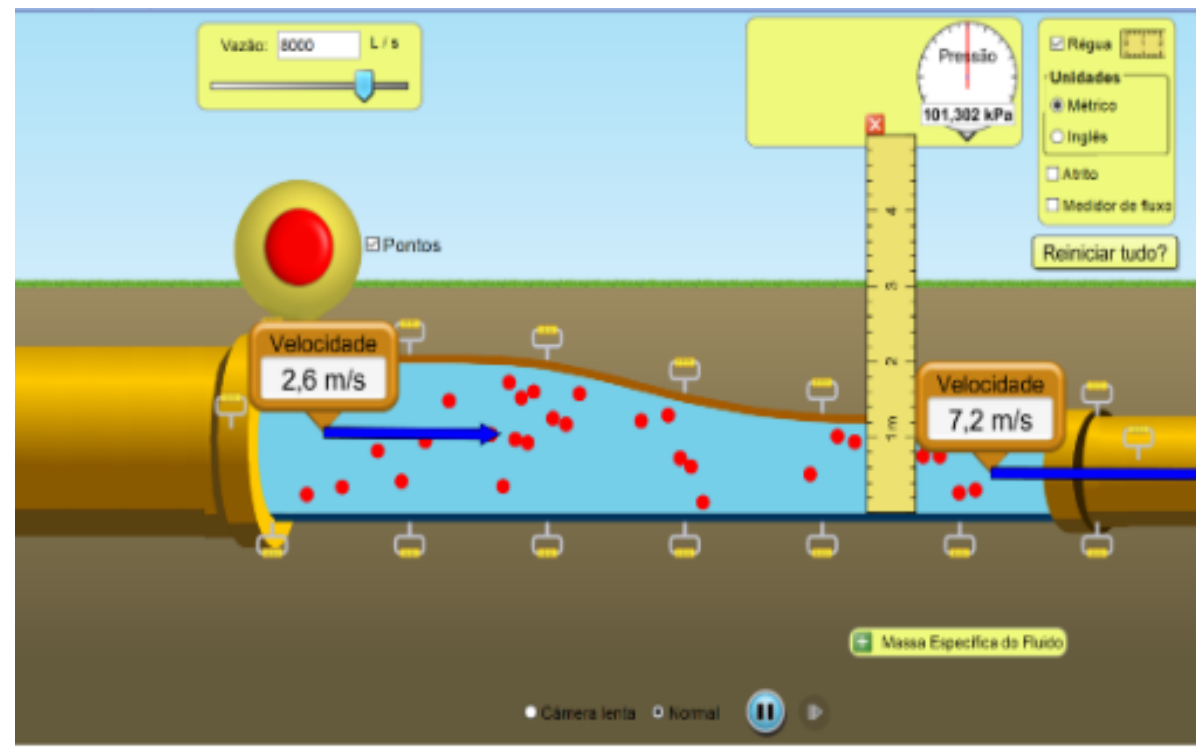

Fonte: acervo pessoal, 2019.

No Quadro 2 são apresentados os cálculos e comentários realizados pela dupla.

Quadro 2 - Cálculos e comentários da dupla de estudantes sobre simulação apresentada na Figura 3

\begin{tabular}{|l|l|l|}
\hline \multicolumn{1}{|c|}{ Dados e Objetivos } & \multicolumn{1}{|c|}{ Cálculos desenvolvidos } & Comentários dos estudantes \\
\hline Objetivo: verificar a validade & Vazão de entrada & $\mathrm{Q}_{1}=\mathrm{Q}_{2}$ \\
da equação da continuidade & $\mathrm{V}_{1}=2,6 \mathrm{~m} / \mathrm{s}$ & Os valores de saída ficaram \\
em regime permanente com & $\mathrm{D}_{1}=2 \mathrm{~m}$ & próximos aos valores de \\
dados selecionados pelo & $\mathrm{A}_{1}=\left(\pi \cdot \mathrm{D}_{1}{ }^{2}\right) / 4$ & \\
estudante. & $\mathrm{A}_{1}=\left(3,14159 \cdot 2^{2}\right) / 4$ & \\
& $\mathrm{~A}_{1}=3,14 \mathrm{~m}^{2}$ & \\
& $\mathrm{Q}_{1}=\mathrm{V}_{1} \cdot \pi \cdot \mathrm{D}_{1}{ }^{2}$ & \\
& $\mathrm{Q}_{1}=\left(2,6 \cdot 3,14159 \cdot 2^{2}\right) / 4$ & \\
& $\mathrm{Q}_{1}=8,168 \mathrm{~m}^{3} / \mathrm{s}$ & \\
& $\mathrm{Vazão} \mathrm{de} \mathrm{saída}$ & \\
& $\mathrm{V}_{2}=7,2 \mathrm{~m} / \mathrm{s}$ & \\
& $\mathrm{D}_{2}=1,20 \mathrm{~m}$ & \\
& $\mathrm{~A}_{2}=\left(\pi \cdot \mathrm{D}_{2}{ }^{2}\right) / 4$ & \\
& $\mathrm{~A}_{2}=\left(3,14159 \cdot 1,20^{2}\right) / 4$ & \\
& $\mathrm{~A}_{2}=1,13 \mathrm{~m}^{2}$ & \\
& $\mathrm{Q}_{2}=\mathrm{V}_{2} \cdot \pi \cdot \mathrm{D}_{2}{ }^{2}$ & \\
& $\mathrm{Q}_{2}=\left(7,2 \cdot 3,14159 \cdot 1,20^{2}\right) / 4$ & \\
& $\mathrm{Q}_{2}=8,143 \mathrm{~m}^{3} / \mathrm{s}$ & \\
\hline
\end{tabular}

Fonte: acervo pessoal, 2019.

Observa-se, por meio dos comentários e do desenvolvimento dos cálculos dos alunos, que os exercícios foram relevantes para a apropriação dos conceitos, e que a integração entre o conteúdo teórico e experimentação virtual na plataforma de simulação 
representou uma importante ferramenta no ensino-aprendizagem de Mecânica dos Fluidos.

\subsection{Avaliação dos estudantes acerca do uso do aplicativo de simulação}

A avaliação da plataforma pelos estudantes foi realizada por intermédio de duas questões qualitativas, sendo a primeira de múltipla escolha e a segunda aberta. Dos 68 estudantes que estavam presentes nas aulas realizadas com a aplicação de simulação, 5 não responderam à avaliação, totalizando 63 respostas válidas e 5 não respondentes.

A primeira questão trazia uma escala com as opções "muito satisfatório", "satisfatório", "regular", "ruim" e "insuficiente", respectivamente, para avaliar a satisfação dos estudantes quanto ao uso do módulo Pressão de um Fluido e Fluxo na aprendizagem dos conceitos introdutórios ao conteúdo de Mecânica dos Fluidos. A maioria dos estudantes avaliou a experimentação por meio da plataforma como muito satisfatória. A Figura 4 representa de forma gráfica o resultado da avaliação da primeira questão.

A segunda questão trazia o enunciado "De que forma a plataforma contribuiu para o seu entendimento do conteúdo teórico estudado?", em que os estudantes poderiam discorrer, sem limite de caracteres, as suas conclusões sobre o desenvolvimento da aula e da sua interação com o aplicativo de simulação. A partir da Análise Textual Discursiva, analisou-se as respostas válidas e emergiram as unidades de significado mais relevantes para o estudo, organizadas nas categorias "Contribuições da tecnologia no processo de ensino-aprendizagem" e "Obstáculos presentes no uso da plataforma", que se referem, respectivamente, às contribuições identificadas nas falas dos estudantes acerca dos aspectos positivos da plataforma, o seu potencial didático para trabalhar o conteúdo de Mecânica dos Fluidos e às dificuldades encontradas no uso da plataforma que, embora identificadas pelos alunos, não se tornaram empecilhos na efetivação da aprendizagem. O Quadro 3 traz as unidades de significados emergentes das falas dos estudantes.

Figura 4 - Avaliação qualitativa dos estudantes sobre o módulo

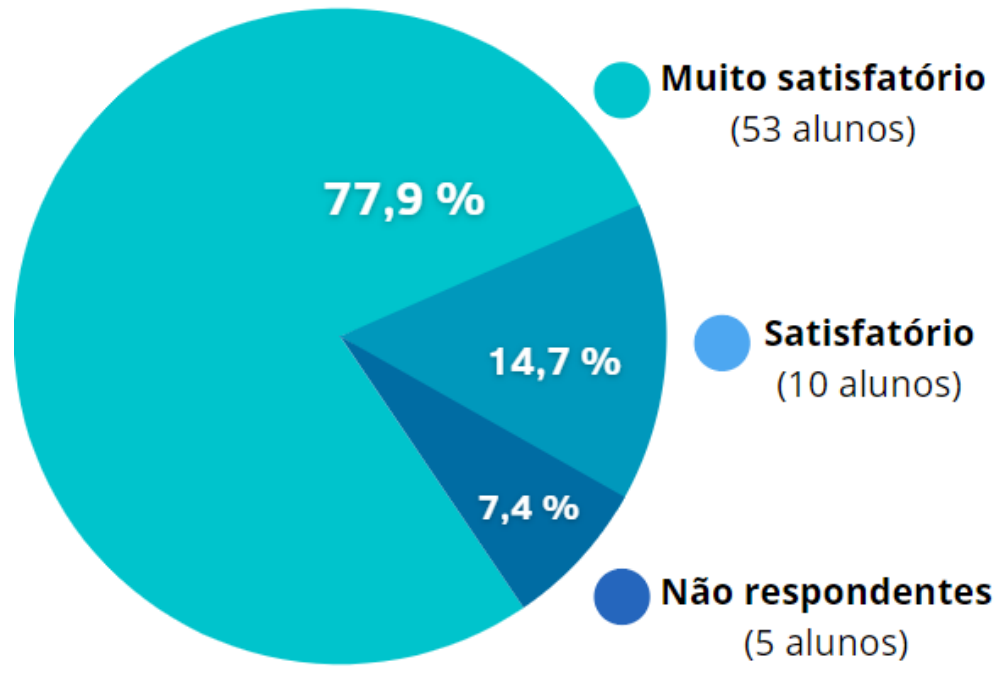

Fonte: acervo pessoal, 2019.

Quadro 3 - Categorias de palavras emergentes a partir da avaliação dos estudantes

\begin{tabular}{|c|c|}
\hline Categorias & Unidades de significado \\
\hline $\begin{array}{c}\text { Contribuições da tecnologia no processo } \\
\text { de ensino-aprendizagem }\end{array}$ & $\begin{array}{l}\text { Convergência entre os conhecimentos teóricos e sua } \\
\text { aplicação real em um sistema simulado. Identificação } \\
\text { de relações positivas entre os exercícios práticos e a }\end{array}$ \\
\hline
\end{tabular}




\begin{tabular}{|c|c|}
\hline & $\begin{array}{c}\text { abstração. Compreensão da influência das variáveis } \\
\text { estudadas no resultado das equações. Uso da } \\
\text { plataforma como um recurso didático que torna a aula } \\
\text { mais dinâmica. }\end{array}$ \\
\hline $\begin{array}{c}\text { Obstáculos presentes na uso da } \\
\text { plataforma }\end{array}$ & $\begin{array}{c}\text { Identificação das imprecisões existentes na plataforma } \\
\text { e das limitações desta, bem como a identificação das } \\
\text { limitações dos equipamentos disponíveis para o o } \\
\text { acesso. }\end{array}$ \\
\hline
\end{tabular}

Fonte: acervo pessoal, 2019.

Como pode ser observado na Figura 4 e no Quadro 3, os estudantes consolidaram o momento como sendo muito relevante para o ensino-aprendizagem em Mecânica dos Fluidos. Durante a aula, todas as duplas aproximaram-se da professora e da aluna de IC. A participação foi superior à observada em aulas tradicionais de resolução de problemas. A própria professora mostrou-se empolgada quando viu a solução de determinadas dificuldades encontradas, como a baixa sensibilidade de alguns mouses, serem propostas pelos próprios estudantes.

As dificuldades encontradas não tiraram a motivação dos estudantes. Chama-se a atenção para uma dupla formada por estudantes que vinham apresentando dificuldades e obtiveram baixo desempenho em avaliações anteriores. Estes mostraram-se empolgados com a visualização dos fenômenos e descreveram as situações observadas nas simulações com uma riqueza de detalhes superior à média da turma, demonstrando compreensão do assunto.

\section{CONSIDERAÇÕES FINAIS}

O presente trabalho objetivou relatar e discutir o uso do aplicativo Pressão de um Fluido e Fluxo, disponibilizado pela plataforma PhET Interactive Simulations (UNIVERSITY OF COLORADO BOULDER, 2021), em experimentações simuladas sobre conceitos de cinemática e estática dos fluidos na disciplina de Operações Unitárias, a partir da avaliação dos estudantes da disciplina e da professora regente acerca das contribuições desta ferramenta para o ensino-aprendizagem de conceitos em Mecânica dos Fluidos.

Com a análise realizada acerca dos dados coletados, concluiu-se que a aplicação de simulação promove uma aprendizagem mais efetiva para os estudantes, uma vez que vai além da abstração teórica e possibilita o contato dos futuros técnicos em Química com sistemas mais concretos. Destacam-se os apontamentos dos estudantes quanto aos pontos de melhoria na plataforma, como a imprecisão dos cálculos, e também a identificação de necessidade de melhorias nos equipamentos, como componentes dos computadores, para favorecer a experiência da simulação. Tais posicionamentos corroboram com a participação ativa e atenta dos estudantes na atividade proposta.

A professora regente da turma também observou o bom desempenho dos estudantes, sobretudo dos que possuem mais dificuldades durante as aulas teóricas, avaliando positivamente a dinâmica. Almeja-se, em momento oportuno, a continuidade do uso do módulo de simulação Pressão de um Fluido e Fluxo, contando com a reelaboração dos exercícios propostos e manutenção da avaliação qualitativa do momento. A busca por outros recursos tecnológicos que possam ser utilizados no contexto educacional para potencializar o desenvolvimento dos alunos e futuros profissionais também será continuada. Devido ao contexto pandêmico, estão sendo desenvolvidas outras estratégias pedagógicas nos demais conteúdos da disciplina, de forma a manter a atualização pedagógica e tecnológica e o objetivo de despertar vocações para a área da engenharia. 


\section{(C) COBENGE E Eventio online

\section{Agradecimentos}

Ao Conselho Nacional de Desenvolvimento Científico e Tecnológico (CNPq), pelo suporte financeiro na forma de bolsa de Iniciação Científica.

\section{REFERÊNCIAS}

ARANTES, Alessandra Riposati et al.. Objetos de aprendizagem no ensino de física: usando simulações do phet. Física na Escola, [S.I.], v. 11, n. 1, p. 27-31, 2010.

AUSUBEL, David Paul. Educational psychology: a cognitive view. 1 ed. New York: Holt, Rinehart, and Winston, 1968. 685 p.

AUSUBEL, David Paul. The acquisition and retention of knowledge: a cognitive view. 1 ed. Dordrecht: Kluwer Academic Publishers, 2000. 210 p.

BARBETTA, Pedro Alberto. Estatística Aplicada às Ciências Sociais. 7 ed.

Florianópolis: Editora da UFSC, 2010. 315 p.

BORDIN, Leandro, BAZZO, Walter Antonio. Essa "tal" filosofia: sobre as concepções de tecnologia e seus reflexos no processo formativo em engenharia. Revista Brasileira de Ensino de Ciência e Tecnologia, Curitiba, v.11, n. 1, p. 228-249, 2018.

BORDIN, Leandro, BAZZO, Walter Antonio. Sobre as muitas variáveis - e incógnitas que se articulam em torno da complexa e não linear relação entre engenharia e vida. Revista Tecnologia e Sociedade, Curitiba, v.13, n. 28, p. 224-239, 2017.

BRASIL. Liane Margarida Rockenbach Tarouco. Ministério da Educação (org.). Objetos de Aprendizagem: teoria e prática. 1. ed. Porto Alegre: Evangraf, 2014. 504 p.

DEMO, Pedro. Educação hoje: "novas" tecnologias, pressões e oportunidades. 1. ed. São Paulo: Atlas, 2009.

FREIRE, Paulo. Pedagogia da esperança: um reencontro com a Pedagogia do oprimido. São Paulo: Paz e Terra, 2006.

HARGREAVES, Andy, FULLAN, Michael. A escola como organização aprendente: buscando uma educação de qualidade. 2 ed. Porto Alegre: Artmed, 2000. 136 p.

MARDEGAN, José Ribeiro. INTEGRAÇÃO DO LABORATÓRIO VIRTUAL "PhET INTERACTIVE SIMULATIONS" NO ENSINO DE FÍSICA. In: XLII Congresso Internacional de Educação e Tecnologias | Encontro de Pesquisadores em Educação a Distância, 2020, São Carlos. Anais. São Carlos. Disponível em:

<https://cietenped.ufscar.br/submissao/index.php/2020/article/view/1607>. Acesso em 15 abril. 2021.

MATURANA, Humberto. Cognição, ciência e vida cotidiana. Belo Horizonte: Editora da UFMG, 2001. $200 \mathrm{p}$

MORAES, Roque. Análise Textual Discursiva. 2. ed. ljuí: Editora Unijuí, 2016. 


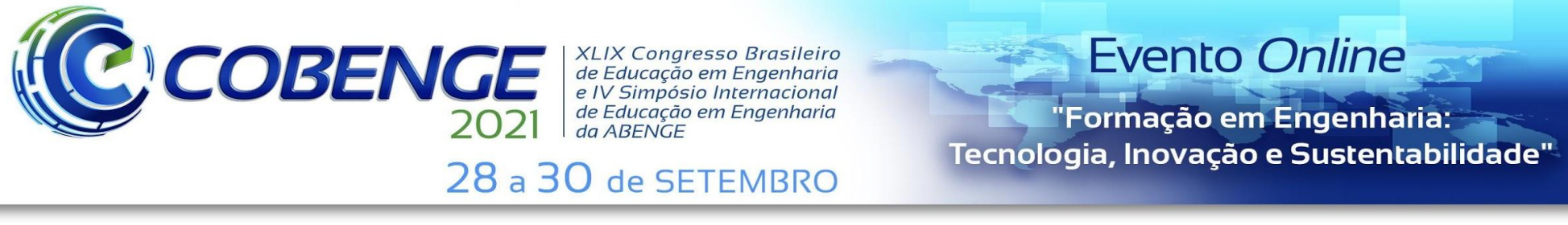

MORAN, José Manuel. A educação que desejamos: Novos desafios e como chegar lá. 5. ed, [s.I.]: Papirus Editora, 2013.

MOREIRA, Marco Antônio. A teoria da Aprendizagem Significativa e sua

implementação em sala de aula. 1 ed. Brasília: Editora da UnB, 2006. 185 p.

NEVES, Rui Gomes; TEODORO, Vítor Duarte. Modelação computacional, ambientes interactivos e o ensino da Ciência, Tecnologia, Engenharia e Matemática. Revista Lusófona de Educação, Lisboa, v. 25, n. 1, p. 35-58, 2013.

PINHEIRO, Denise Marques et al.. PROGRAMAS DE SIMULAÇÃO PODEM AUXILIAR O PROFESSOR EM SALA DE AULA? UM ESTUDO DE CASO DO USO DO PROGRAMA INTERACTIVE PHYSICS APLICADO EM OSCILAÇÕES MECÂNICAS. In: XL Congresso Brasileiro de Educação em Engenharia. Anais. Belém. Disponível em:<http://www.abenge.org.br/cobenge/legado/arquivos/7/artigos/103045.pdf>. Acesso em 15 abril. 2021.

SCHAFFEL, Izabela de França et al.. Análise dos estilos de aprendizagem de alunos do Ensino Técnico na disciplina de Operações Unitárias. Debates em Educação, Maceió, v. 11, n. 24, p. 432-452, 2019.

SCHIMITT, B.; SCHEIN, Z P. ESTUDO DO APPLET "FORÇAS E MOVIMENTO", DISPONIVVEL NA PLATAFORMA PhET INTERACTIVE SIMULATIONS, PARA O ENSINO DE FÍSICA. Form@ção de Professores em Revista, Taquara, v.1, n. 2, p. 34-52, 2020.

SILVA, Swéle Rachel da; MELO, Cláudia Adriana de Sousa. A UTILIZAÇÃO DA SIMULAÇÃO "FORÇA E MOVIMENTO" DA PLATAFORMA PhET, COMO RECURSO DIDÁTICO NO PROCESSO DE ENSINO-APRENDIZAGEM NO ENSINO MÉDIO.

Revista Educação e Emancipação, São Luís, v. 9, n. 2, p. 257-277, 2016.

UNIVERSITY OF COLORADO BOULDER (Estados Unidos). PhET Interactive

Simulations: Simulações Interativas para Ciência e Matemática. [S. I.], 2021. Disponível em: <https://phet.colorado.edu/pt_BR/>. Acesso em: 15 abr. 2021.

UNIVERSITY OF COLORADO BOULDER (Estados Unidos). Pressão de um Fluido e Fluxo. [S. I.], 2021. Disponível em:

$<$ https://phet.colorado.edu/sims/cheerpj/fluid-pressure-and-flow/latest/fluid-pressure-and-fl ow.html?simulation=fluid-pressure-and-flow\&locale=pt_BR>. Acesso em: 15 abr. 2021.

\section{USE OF THE PHET SIMULATION PLATFORM IN THE TEACHING-LEARNING PROCESS OF FLUID MECHANICS IN THE TECHNICAL COURSE IN CHEMISTRY}

Abstract: This article analyzes and discusses the use of a simulation application in the teaching of Fluid Mechanics, content of the menu of the discipline of Unit Operations, taught in the fourth period of a technical course in Chemistry concomitant with the Federal Network. The work is the result of the continuity of the research developed in a Scientific 
Initiation project, started with the identification of students' learning styles and continued to implement pedagogical strategies capable of contemplating different profiles. From the search and studies of the research group, we selected the Pressure of a Fluid and Flow module, available on the free PhET Interactive Simulations platform, using two simulations: fluid pressure in static systems and flow with constant flow in a cylindrical duct. The students were evaluated for performance in closed questions proposed with the use of the platform, in which they were able to explore the measurement tools, changing properties of the fluid and systems, performing simulations, calculations, analyzing and commenting on the effects observed in different situations. They were also offered a qualitative assessment of the platform through open-ended questions. From the Discursive Textual Analysis, the categories of technology contributions in the teaching-learning process and pedagogical obstacles present in the application emerged from the students' speeches. In these, students' performance, their opinions about the use of the platform and contributions to learning were discussed, highlighting the convergence between theoretical knowledge and its application in a simulated system and the application as a didactic resource that makes it the most dynamic class.

Keywords: Simulation. Teaching-Learning. Unitary Operations. 